\title{
Collaborations, évitements et conflits entre disciplines autour d'un terrain partagé
}

\author{
Clémence Massart \\ Sociologue, Université de Liège, Département des Sciences et gestion de l'environnement, Unité Seed, 6700 Arlon, Belgique
}

\begin{abstract}
Cet article rend compte d'une enquête sociologique auprès de scientifiques de plusieurs disciplines qui ont eu des difficultés à travailler en inter ou multidisciplinarité, alors que tout pouvait y mener : un objet commun, la maladie de Lyme, et une entité spatiale commune, la forêt de Sénart. On connaît le risque des biais analytiques dans un contexte d'asymétrie de positions des différents protagonistes, comme on sait l'influence des mondes académique et institutionnel où les critères d'évaluation et les espaces de compilation des savoirs sont très différents. De fait, ce terrain n'a pu être "partagé » car, investit suite à une opportunité externe de financement, il n'a pas produit suffisamment d'éléments inattendus pour déstabiliser les routines et les mondes d'appartenance des agents engagés dans les diverses configurations. D'où une communauté largement fictive - ainsi que des rivalités qui ne le sont peut-être pas moins -, chacun entrant dans le projet avec ses propres questions et objectifs.
\end{abstract}

La Rédaction

\section{Mots-clés :}

santé ; environnement ; espace-frontière ; interdisciplinarité ; maladie de Lyme

\section{Keywords:}

health;

environment;

border zone;

interdisciplinarity;

Lyme disease
Résumé - Cet article interroge les collaborations, les conflits et les évitements qui se nouent entre disciplines autour d'un terrain d'étude partagé. À l'aide de la notion d'espace-frontière, j'analyserai l'exemple de la forêt périparisienne de Sénart, investie par des chercheurs qui travaillent sur la maladie de Lyme et son vecteur, des tiques du genre Ixodes. Pour les sciences qui fondent leurs hypothèses sur l'observation du terrain et l'interprétation d'indices, telles que l'écologie, la géographie ou encore l'épidémiologie, Sénart constitue un site d'exception. Elle combine en effet tous les facteurs de risque actuellement identifiés comme favorisant le maintien et le développement de cas de maladie de Lyme. Trop nombreux, ces facteurs échappent à la compréhension d'un unique chercheur ou d'une unique communauté scientifique. Pour appréhender conjointement ces facteurs de risque, des collaborations se sont nouées autour de Sénart par l'intermédiaire de projets de recherche. Mais cet espace collaboratif fait l'objet de conflits, de concurrence ou, plus simplement, de critiques au sein des équipes de recherche qui y travaillent. J'analyserai les relations nouées et les formes d'apprentissage permises par cet espace partagé. Je montrerai notamment que les chercheurs conçoivent la représentativité des études de manière différente en fonction de leur pratique du terrain. Les relations entre acteurs semblent finalement déterminées par trois types de conditions imbriquées : déontologiques, épistémiques et institutionnelles.

\begin{abstract}
Collaborations, avoidance and conflict between disciplines around a shared field. Collaboration, avoidance and conflict between disciplines around a shared field. This article addresses collaborations, conflicts and avoidances that develop between disciplines over a shared field of study. In this perspective and using the notion of border zone (Kohler, 2001 and 2011), I analyzed the Sénart forest on the outskirts of Paris, the study area of researchers working on Lyme disease and its primary vector, ticks of the Ixodes genus. For sciences such as ecology, geography or epidemiology that base their assumptions on field observation and the interpretation of indices, Sénart is an exceptional site. It combines all the risk factors currently identified as favoring the persistence and development of Lyme disease cases. However, these various factors and their combinations exceed the scope of a single scientific community or a single researcher. To investigate the risk factors. interdisciplinary work developed on the Sénart case through common research projects. However, instead of remaining mere collaborative work, the project turned out to be a source of conflicts, competition and critics within the research teams involved. These issues still need addressing. In this paper I discuss the kinds of relationships and the various learning processes enabled by this shared space. I show in particular that researchers view study representativeness differently depending on their field practice. To conclude I show that the relations between these various actors seem to be determined in fine by three types of nested conditions: institutional, cognitive, and ethical.
\end{abstract}

Auteur correspondant : C. Massart, clemence.massart@ulg.ac.be 


\section{La maladie de Lyme}

La borréliose de Lyme est une maladie bactérienne infectieuse, émergente et complexe. Elle se transmet par l'intermédiaire d'une tique du genre Ixodes (en France, Ixodes ricinus) qui se nourrit sur la faune sauvage réservoir des pathogènes (des bactéries du genre Borrelia). Prédominante dans l'hémisphère nord, la borréliose de Lyme est donc une maladie vectorielle mais aussi zoonotique puisqu'elle passe de plusieurs espèces animales réservoir à l'homme.

Lyme illustre un type de maladie délaissé par la sociologie de l'action publique, dans le sens où ce problème de santé publique en train de se construire est controversé, de manière discrète mais croissante (Gilbert et Henry, 2012 ; Massart, 2013). Plus de 40 ans après sa mise en évidence aux États-Unis, de nombreuses incertitudes subsistent en effet à son égard. Sur le plan clinique, l'hypothèse d'une évolution chronique est avancée par de nombreux patients mais niée par la conférence de consensus française qui a eu lieu en 2006. Particulièrement aiguë outreAtlantique, la controverse entre les médecins et les patients s'immisce actuellement en Europe, sans toutefois bénéficier d'un traitement médiatique aussi marqué.

Aux États-Unis comme en Europe, la controverse se déploie d'abord autour des tests diagnostics : calibrés sur l'hypothèse d'une maladie rare (ne pouvant concerner plus de $5 \%$ des donneurs de sang), ils ne détecteraient que les malades ayant produit de nombreux anticorps. Conçus pour détecter un unique pathogène, ils échoueraient également à identifier de potentielles co-infections (or Ixodes ricinus peut transmettre simultanément de très nombreux pathogènes dont les interactions sont méconnues). Enfin, seules les souches réputées nocives seraient prises en compte, sans que l'effet des autres souches ait fait l'objet d'études systématiques. Sur la base de ces critiques, les patients mettent en cause l'efficacité des traitements : détectée trop tard, la maladie ne serait pas éradiquée par les huit semaines d'antibiotiques prescrits. Elle évoluerait dès lors vers une forme chronique, marquée par la dissémination des borrélies (les bactéries responsables de la maladie de Lyme) dans l'organisme. Pour combattre cette forme chronique, les patients réclament des traitements plus longs et plus individualisés.

L'épidémiologie de la maladie soulève également de nombreux débats entre les communautés scientifiques (entomologistes, écologues, microbiologistes, etc.). Comme en témoignent les diagnostics de plus en plus fréquents, les tiques semblent se propager dans les régions méditerranéennes d'où elles étaient réputées absentes. Aucune étude récente n'est venue réinterroger la seconde affirmation, produite par la littérature entomologique (Fontenille et al., 2013) et régulièrement mise en doute. Plus globalement, si les tiques peuvent potentiellement s'implanter dans tous les espaces naturels tempérés, leur répartition spatiale présente des variations encore mal comprises. Celles-ci seraient entre autres liées à l'implantation des espèces animales et à leur rencontre. Les espèces animales accessibles aux tiques déterminent également le portage en pathogènes de ces dernières puisque chaque espèce véhicule des souches différentes de borrélies en quantité variable (mais aussi d'autres pathogènes). Par conséquent, en fonction de la diversité et de la densité des réservoirs présents, la prévalence de la maladie peut varier sur le plan géographique et écologique et, avec elle, le risque de transmission, qui est également lié à la fréquentation des milieux naturels par les hommes.

Pour étudier ces variations, les chercheurs doivent sélectionner des milieux non pas tant représentatifs que spécifiques, dans le sens où ils combinent un ensemble de paramètres suspectés influencer la densité de tiques et leur taux d'infection. La forêt de Sénart, qui sera envisagée dans cet article, représente un de ces milieux singuliers dans lesquels circulent de très nombreux réservoirs, où les tiques sont donc abondantes et largement infectées, mais où les promeneurs sont aussi très nombreux. Sénart constitue en cela une forêt modèle, particulièrement attractive pour les sciences qui procèdent par observation et interprétation d'indices ${ }^{1}$.

\section{La forêt de Sénart : un site de contiguïté risqué}

\section{Sénart, site remarquable}

La forêt de Sénart est une forêt domaniale de 32000 hectares. Située à 30 kilomètres du centre de Paris, Sénart est l'exemple type des forêts périurbaines, enclavées dans une zone très densément urbanisée. Dans ces forêts, les risques de transmission d'agents infectieux sont non négligeables, les usages y étant aussi nombreux et divers que les usagers. Par sa situation géographique et ses aménagements, Sénart attire en effet de nombreux visiteurs : elle est traversée de chemins de randonnée, auxquels mènent cinq parkings. Outre les «promeneurs du dimanche », on y rencontre des randonneurs expérimentés (associations), des cueilleurs de champignons et de jonquilles, voire des publics plus marginaux (prostituées, homosexuels).

La forêt de Sénart réunit également une grande diversité d'espèces animales. Le grand gibier est si abondant

\footnotetext{
1 J'ai réalisé cette recherche dans le cadre de ma thèse de doctorat en sociologie (UPMF - Ulg). J'ai mené des entretiens avec tous les acteurs mentionnés dans cet article et effectué une récolte de tiques (avec les écologues du MNHN) ainsi que la visite d'un élevage de tiques.
} 
sur ce territoire de chasse que la régulation cynégétique est nécessaire pour éviter les accidents de la route. Sénart est aussi riche en micromammifères et notamment en tamias (Tamias sibiricus barberi), un écureuil originaire d'Asie. Vendus dans les animaleries, les tamias qui se sont échappés ou ont été relâchés par leurs propriétaires sont désormais bien implantés dans certaines forêts françaises. C'est le cas de Sénart, où les effectifs sont particulièrement anciens (années 1970) et abondants.

La forêt de Sénart offre également aux chercheurs des conditions de travail idéales. Situés dans le parc de la Faisanderie, les locaux de l'Office national des forêts (ONF) leur assurent un confort optimal : ils peuvent y déjeuner, travailler en attendant que des tamias soient pris dans les pièges, entreposer du matériel, mais aussi bénéficier des conseils et de la connaissance du lieu qu'a le garde ONF qui réside sur place. Proche de Paris, la forêt de Sénart économise également aux chercheurs des déplacements coûteux en temps, et qui peuvent altérer le matériel biologique, donc le résultat des récoltes de tiques comme des captures d'animaux.

Par la coprésence de très nombreux vivants (publics, réservoirs sauvages, pathogènes, vecteurs) qui concentrent le risque, mais aussi par les facilités matérielles qu'elle procure, la forêt de Sénart a été choisie par plusieurs chercheurs pour servir de support à la qualification de la maladie de Lyme. Ce lieu singulier permet en effet d'envisager le risque sous tous les angles : des études sociogéographiques visant à évaluer celui issu des pratiques humaines comme des études microbiologiques du matériel récolté, ou encore, eu égard à la variété des hôtes réservoir, des études relatives à la spécialisation alimentaire des populations de tiques peuvent être menées. Par ailleurs, Sénart se distingue d'autres forêts périurbaines par la présence du tamia, dont les charges en tiques sont étonnamment élevées et qui est aujourd'hui suspecté d'être un excellent réservoir des borrélies.

Comme le souligne Kohler (2002), si la spécificité des sciences de laboratoire est de créer des espaces génériques, indépendants du contexte et transposables en tous lieux (placeless place), dont elles tirent d'ailleurs leur crédibilité, celle des sciences de terrain est de choisir avec intelligence un espace singulier porteur d'indices parlants qui permettent des comparaisons avec d'autres lieux. Les chercheurs qui ont investi Sénart ont fait preuve $\mathrm{d}$ 'intelligence en choisissant un site dont la singularité permet d'éclairer particulièrement certaines questions relatives à l'épidémiologie de la maladie de Lyme.

\section{Historique des collaborations : \\ le tamia comme " super réservoir " et l'augmentation des borrélioses autour de Sénart}

La forêt de Sénart a d'abord été investie par un écologue dynamicien des populations et un écologue parasitologue, tous deux rattachés au Muséum national d'histoire naturelle (MNHN). Depuis 2003, ils y étudient l'écologie du tamia. En capturant des tamias, les écologues du Muséum ont été frappés par leur charge en tiques. "La première surprise avec le tamia, c'est qu'il avait énormément de tiques. Par exemple, le campagnol roussâtre ou le mulot sylvestre ont peu de tiques, une dizaine maximum. Et le tamia, c'était des centaines». Forts de ce constat, ils se sont intéressés aux tiques et aux pathogènes qu'elles peuvent transmettre. En effet, à partir du moment où les tiques étaient retrouvées en masse sur les tamias, ces derniers pouvaient devenir des réservoirs potentiels pour les pathogènes contenus dans les tiques $^{2}$. Cette hypothèse était étayée par le fait que le tamia strié (Tamia striatus), localisé sur le continent américain, est reconnu comme un excellent réservoir de borrélies $^{3}$ : « Il est tout à fait possible que, si le tamia strié est un réservoir important, son espèce soeur, le tamia de Sibérie, le soit aussi » (écologue du MNHN).

Identifier le tamia à un réservoir permet à ces écologues de l'associer aux espèces invasives, problématique qui marque leur carrière : "Une espèce invasive, c'est généralement une espèce introduite qui a un impact soit écologique, soit économique ou sanitaire ${ }^{4} »$. L'impact économique du tamia semblant nul et son impact écologique délicat à prouver, c'est sur l'impact sanitaire qu'ils se concentrent. La piste des maladies à tiques s'est d'autant plus vite révélée intéressante que les deux écologues y ont vu une opportunité en matière de positionnement scientifique. Les exemples «d'espèces introduites qui ont des conséquences sur le plan de la santé humaine »étant rares, l'impact sanitaire des tamias ouvre un champ de questionnements peu abordés et propices à l'obtention de crédits de recherche : «Pour nous, c'est relativement intéressant parce qu'on arrive par ce biais là à avoir des financements ».

Par l'intermédiaire d'amis communs, l'un des écologues a rencontré une écoépidémiologiste qui dirige le laboratoire Epia (Inra) et s'est spécialisée dans l'étude des

\footnotetext{
2 Les animaux sur lesquels se nourrissent les vecteurs peuvent aussi être des culs-de-sac épidémiologiques, c'est-à-dire qu'ils ne multiplieront pas les pathogènes. Cen'est toutefois pas l'hypothèse qui a été privilégiée par les acteurs mentionnés dans cet article.

3 Par contre, lorsqu'on encode sur Google Scholar ou Scopus "Tamia sibiricus » et "Lyme », seuls des articles auxquels ont participé les écologues du MNHN et du laboratoire Epia (Inra) apparaissent.

4 Les chercheurs se réfèrent ici à la définition que l'Union internationale pour la conservation de la nature (UICN) donne d'une espèce invasive, plus précisément qualifiée d'espèce exotique envahissante. Cette définition ne s'assimile pas à la simple introduction d'une espèce non indigène. L'UICN précise en effet que les espèces invasives ont des impacts négatifs soit sur la santé, soit sur les écosystèmes, soit sur l'économie.
} 


\section{Encadré. L'accès au terrain}

Dans le cadre de ma thèse de doctorat, j'ai réalisé des entretiens avec les acteurs cités dans l'article. Tous ont été informés de ma question de recherche et de l'usage que je ferai des données (je travaillais sur la manière dont chacun produisait des connaissances sur la maladie de Lyme, son vecteur et ses réservoirs, ainsi que sur les modalités de rencontre entre ces savoirs). Certains ont eu l'occasion d'entendre mes résultats, dans le cadre du groupe de recherche « Tiques et maladies à tiques » (TMT) dont je suis membre, ou dans des séminaires qu'ils ont organisés à mon intention. Tous ont reçu un exemplaire numérique de ma thèse et certains ont commenté mon travail.

Je suis entrée en contact de manière différente avec les acteurs en fonction de leurs disponibilités. J'ai passé une journée avec les écologues du Muséum national d'histoire naturelle (MNHN) sur le site de Sénart pour observer leurs récoltes de tiques et leurs captures de tamias. J'ai rencontré les membres du CNR à l'Institut Pasteur de Paris où j'ai pu « visiter » leur élevage de tiques. J'ai réalisé un entretien téléphonique avec l'écoépidémiologiste du laboratoire Epia où je suis allée présenter mes travaux de thèse en 2014. Je l'ai aussi rencontrée à plusieurs reprises lors des réunions du groupe TMT, qui réunit les membres du CNR, du MNHN et du laboratoire Bipar. J'ai rencontré les membres du laboratoire Bipar et j'ai réalisé un entretien téléphonique avec l'infectiologue de Villeneuve SaintGeorges. J'ai eu deux entretiens téléphoniques avec le géographe du Ladyss : une première fois durant ma thèse, une deuxième fois après qu'il ait lu mon manuscrit. Nous avons discuté à cette occasion des analyses que j'avais produites dans le chapitre dont est tiré cet article. Enfin, j'ai analysé le forum lié à l'association France Lyme et j'ai assisté à une journée de sensibilisation à la maladie de Lyme.

tiques et de leurs pathogènes. Celle-ci, intéressée par ses observations relatives au tamia, en a discuté avec un médecin spécialiste de Lyme à l'Institut de veille sanitaire (InVS), qui voyait au même moment s'accroître les déclarations de maladies aux alentours de Sénart. Par l'intermédiaire de l'InVS, le Centre national de référence (CNR) des Borrelia s'est intéressé à la problématique des tamias et a choisi la forêt de Sénart pour qualifier le risque en Île-de-France. C'est également grâce à l'InVS qu'un géographe du laboratoire Dynamiques sociales et recomposition des espaces (Ladyss) a été mis en contact avec les autres chercheurs. La rencontre entre l'écologue du Muséum et l'écoépidémiologiste d'Epia a donc favorisé le rapprochement entre deux observations - la charge en tiques des tamias et l'augmentation des déclarations autour de Sénart - et finalement conduit un ensemble de scientifiques à s'interroger sur la fonction de réservoir du tamia.

\section{La convergence des laboratoires autour d'un site remarquable}

Parce qu'elle concentre des facteurs de risque particulièrement affirmés, qu'elle accueille une ancienne et abondante population de tamias, que le rôle de réservoir de ces derniers est suspecté eu égard à leur impressionnante charge en tiques et à l'augmentation des cas de maladie de Lyme, Sénart est devenue un espace-frontière, capable d'intéresser plusieurs acteurs répondant chacun à leurs propres questions.

La notion d'espace-frontière (border zone) a été élaborée en histoire des sciences par Kohler (2002, 2011). Elle s'inscrit dans un ensemble de travaux initiés à partir du concept d'objet-frontière (boundary object, Star et Griesemer, 1989) et de ses différentes déclinaisons: organisationfrontière (Guston, 2001), concept intermédiaire (Teulier et Hubert, 2008) et objet intermédiaire (Mélard 2008; Trompette et Vinck, 2009). Kohler a utilisé cette notion pour décrire les relations entre science de terrain et science de laboratoire, en montrant qu'entre elles existait une zone d'échange poreuse et dynamique plutôt qu'une barrière étanche et statique. Dans les espaces-frontières circulent des scientifiques, des objets et des idées. Des pratiques nouvelles et hybrides peuvent en résulter, mais également des frictions.

L'espace-frontière est fréquemment mobilisé pour décrire les conditions et les limites des collaborations entre acteurs, qu'ils s'agissent de chercheurs, de gestionnaires ou d'usagers (Granjou, 2013). Les espacesfrontières engageraient un apprentissage mutuel, luimême source d'innovation : l'espace d'échange instauré ferait évoluer le cadrage donné à une problématique en même temps que l'identité des protagonistes impliqués. La notion a souvent été appliquée aux collaborations nouées au sein des sciences du vivant, entre modélisateurs écologiques et naturalistes de terrain par exemple (Mauz et Granjou, 2013). Je m'inscrirai dans cette perspective, mais j'envisagerai la notion de manière très littérale, en l'ancrant dans un espace singulier.

Aujourd'hui, six équipes se sont investies dans l'étude de la circulation de Lyme en forêt de Sénart ( $c f$. Encadré pour les modalités de rencontre avec ces acteurs).

L'UMR 7204 (MNHN - CNRS) est représentée par les deux écologues spécialistes des tamias déjà cités qui récoltent des tiques et des micromammifères pour leurs partenaires. 
Installé à l'Institut Pasteur jusque fin 2011, et à ce jour recentré à Strasbourg, le CNR des Borrelia détermine les tiques et analyse leur portage en borrélies ${ }^{5}$. Les missions du CNR incluent l'étude épidémiologique du risque de maladie de Lyme sur toute la France. Concrètement, par département, le CNR combine les déclarations de médecins (ce qu'ils appellent le « risque de maladie » puisqu'il renvoie au nombre de personnes infectées) à l'analyse du portage des bactéries par les tiques (ce qu'ils appellent le « risque acarologique ", par analogie au «risque entomologique » plus couramment utilisé en matière de transmission vectorielle, et qu'ils appréhendent par les récoltes de tiques).

Le laboratoire Epia (Inra) a une expertise en matière d'épidémiologie associant l'analyse des tiques et celle des mammifères, réservoirs des pathogènes et hôtes des tiques. Dans le cadre des études sur Sénart, Epia biopsie les oreilles de micromammifères récoltées par les écologues. Les membres du laboratoire tentent également d'identifier les déplacements des animaux pour mieux prévoir les zones à forte densité de tiques.

Le laboratoire Bipar (Inra - École vétérinaire de Maison Alfort) est composé de parasitologues et de microbiologistes spécialistes des bactéries. Il possède un élevage de tiques, outil nécessaire pour réaliser la phase expérimentale, qui consiste à gorger une tique saine sur un tamia infecté puis sur un animal sain afin d'observer si les bactéries s'y développent et, auquel cas, conclure que le tamia est réservoir avec une compétence estimée.

$\mathrm{Au}$ sein du Ladyss (laboratoire Dynamiques sociales et recomposition des espaces) - UMR 7533, travaille un géographe qui tente de corréler la présence des tiques infectées aux parcours des usagers de Sénart et aux milieux qu'ils privilégient. L'association de malades France Lyme, qui porte la position des patients dans la controverse médicale, est associée à ce géographe par l'intermédiaire d'un projet de recherche déposé dans le cadre du programme Partenariats institutions-citoyens pour la recherche et l'innovation (Picri $\left.{ }^{6}\right)$ et consacré à la borréliose de Lyme.

Le CHU de Villeneuve Saint-Georges compte un infectiologue actif depuis plusieurs années dans la problématique de la maladie de Lyme. Il fournit des sérums de malades qui ont fréquenté la forêt de Sénart et couvre donc l'émergence des cas et du diagnostic.

\footnotetext{
5 Créé en 2002, le CNR des Borrelia s'est installé à l'Institut Pasteur dans le laboratoire d'une entomologiste médicale pionnière dans l'étude des Ixodes ricinus et de leurs pathogènes (elle a réalisé les premières études épidémiologiques au début des années 1980, peu après que la maladie ait été détectée aux ÉtatsUnis). Un CNR associé avait été créé à l'Institut de bactériologie de Strasbourg. Celui-ci s'occupait des aspects microbiologiques tandis que le CNR de Pasteur prenait en charge les dimensions entomologiques et épidémiologiques. Depuis 2012, le CNR de Strasbourg est devenu le seul CNR dédié aux borrélioses.
}

Chacune de ces équipes s'interroge sur un aspect particulier qui conduit à l'estimation du risque de maladie de Lyme en forêt de Sénart. Pour évaluer ce risque, il s'agit de déterminer les facteurs (ou aléas) qui l'amplifient, et qui peuvent concerner autant la dynamique d'une population animale, que les préférences des usagers de la forêt (qui les exposent plus ou moins à l'infection). Les questions posées pourraient donc converger, puisqu'aucune de ces équipes n'effectue exactement le même travail que les autres, et que la multiplicité générale des facteurs responsables de l'infection rend la multidisciplinarité nécessaire. Ce n'est pourtant pas le cas et les équipes citées sont associées autour de deux configurations de recherche distinctes.

Ces deux configurations de recherche présentent trois particularités que je vais détailler dans la section suivante. Tout d'abord, une tension forte est apparue entre deux équipes qui n'échangent aucune information. En raison de cette mésentente, deux projets sont en cours, les collaborations n'ayant pu inclure l'ensemble des acteurs dans un même projet, ainsi que cela avait été initialement prévu dans le cadre de la réponse à un appel d'offre de l'Agence nationale de la recherche (ANR). Ensuite, malgré les frictions mentionnées précédemment et parce qu'il bénéficie d'une position institutionnelle privilégiée, le CNR participe aux deux projets et s'inscrit donc dans les deux configurations. Enfin, au sein de chaque projet, des tensions se manifestent. Elles relèvent de la manière dont est conçue la représentativité des résultats scientifiques issus des relevés de terrain, mais aussi des conflits noués antérieurement dans d'autres espaces. Pratique centrale dans l'étude du risque en forêt de Sénart, la récolte de tiques laisse voir le degré de conflit et de collaboration qui s'est instauré entre les acteurs.

Je commencerai par expliquer pourquoi l'ensemble des équipes n'a pu s'articuler autour du risque de maladie de Lyme en forêt de Sénart. Des critères déontologiques ont en effet limité la capacité frontière de Sénart et engendré deux configurations de recherche, dont les questions sont connexes mais qui opèrent en parallèle sur un même espace. Je montrerai qu'une des deux configurations apparaît plus précaire, dans le sens où elle réunit essentiellement des acteurs que je qualifierai d' «outsiders » selon Becker (1963), c'est-à-dire peu insérés dans les autres réseaux de recherche dédiés à la maladie de Lyme.

\footnotetext{
6 Financés par la région Île-de-France (Conseil régional), les programmes Picri ont pour particularité d'exiger la participation de citoyens concernés. Ils sont organisés autour de domaines d'intérêt majeur (DIM) parmi lesquels figure l'axe "maladies infectieuses, parasitaires et nosocomiales émergentes » (Malinf), programmé pour une durée de quatre ans à partir de 2011 et coordonné par l'Institut Pasteur. Le projet Picri conçu autour de la maladie de Lyme se rattache à l'axe zoonose émergente non parasitaire.
} 
Cet isolement se note sur un plan méthodologique : la récolte des tiques s'effectue selon un protocole qu'aucun autre acteur ne mobilise et le rôle du tamia est laissé de côté au profit de l'étude plus classique des chevreuils. Je détaillerai ensuite comment interagissent les acteurs au sein des deux configurations. Il s'agira de souligner les facteurs d'apprentissage qui permettent de considérer que Sénart opère comme un espace-frontière, mais également les facteurs qui limitent, au sein des projets de recherche, l'apprentissage et l'hybridation des savoirs.

\section{Une double configuration de recherche}

\section{La maladresse comme limite aux espaces-frontières}

Lorsque la collaboration s'est établie entre des membres du MNHN, d'Epia et du CNR, la spécialiste de la maladie de Lyme de l'InVS avait envisagé d'y associer un géographe du Ladyss récemment intéressé par la problématique de Lyme. Celui-ci devait déterminer les parcours des usagers de la forêt, ce qui permettrait d'estimer l'exposition des individus. En combinant la densité de tiques, de réservoirs et de promeneurs ainsi que leurs probabilités de rencontre (qui déterminent le risque de transmission), il deviendrait possible d'établir une prévention fine dans le secteur de Sénart. Une première réponse à l'appel d'offre de l'ANR a été conçue dans ce sens et déposée par l'ensemble de ces équipes. Cela leur a permis de mutualiser leurs questions, mais le projet n'a pas été retenu.

Suite à cette première tentative d'obtention de financements, la collaboration entre les écologues du MNHN et le géographe du Ladyss a avorté pour des questions de confiance et de déontologie scientifique. Une doctorante travaillant avec le géographe a en effet conçu un projet de thèse en s'inspirant des comptes rendus des discussions collectives conduites pour répondre à l'appel d'offre de l'ANR, sans en informer les autres participants. Estimant qu'entamer une collaboration implique de partager ses idées avec les autres membres sans que ceux-ci les récupèrent à leur compte, les écologues ont perçu cette démarche comme une rupture du contrat de confiance qui conditionnait la collaboration. « Dans la situation du tamia, comme j'ai initié le truc, j'ai choisi les collaborations que je voulais. Donc toutes les collaborations vont merveilleusement bien parce que c'est des gens choisis. Il y en a d'autres qui ont voulu collaborer, que je ne sentais pas et avec qui on n'a pas collaboré » (écologue du $\mathrm{MNHN}$ ).

Les relations se sont encore envenimées à partir du moment où le géographe a présenté son propre projet de recherche et obtenu des financements via le programme Picri octroyé par la région Île-de-France, qui finance également les études des écologues en forêt de Sénart. Le géographe a répondu à cet appel à projet sur les maladies émergentes en proposant une carte qu'il appelle « carte $\mathrm{du}$ risque », bien que celle-ci se distingue du risque au sens épidémiologique, puisqu'elle est établie à partir de la charge en réservoirs et en tiques des différents groupements forestiers. Il a donc conçu un projet quasiment identique à celui des écologues dans ses objectifs et certaines de ses collaborations. Les écologues y ont répondu par une sanction immédiate et probablement définitive : le refus de toute collaboration avec lui et ses partenaires.

\begin{abstract}
«Il y avait une liste des partenaires qui étaient impliqués dans le Picri et puis des partenaires susceptibles de nous rejoindre. J'ai remis les gens qui devaient travailler avec nous dans l'ANR parce que je pensais qu'on allait travailler avec eux, même si on était sur des financements différents. Et je pense que l'écologue du MNHN m'en veut beaucoup de l'avoir mis dans les gens susceptibles de nous rejoindre sans lui avoir de prime abord demandé son avis. Ça fait que maintenant, il y a un différend entre nous parce que j'ai été maladroit à un moment donné » (géographe du Ladyss).
\end{abstract}

La tension s'étend désormais à leurs collaborations respectives. Le géographe ne participe pas au groupe pluridisciplinaire «Tiques et maladie à tiques » (TMT), qui réunit l'essentiel des chercheurs français travaillant sur les tiques et auquel participent les écologues du MNHN, Bipar, Epia et le CNR. En retour, les écologues du MNHN contribuent à discréditer France Lyme, qui s'est associée au géographe, en prétextant que l'association s'alimente de catastrophisme et de subjectivité. Il semble donc que la méfiance des écologues envers le géographe et sa doctorante s'est simplement étendue à l'association avec laquelle ces derniers collaborent et eux-mêmes refusent d'entretenir tout contact : « À tout moment France Lyme me contacte en disant "Est-ce qu'on peut avoir vos résultats sur le tamia ?" Non, je ne veux pas. Attendez les publications » (écologue du MNHN).

«La rétention est le fait d'un accord entre l'Institut Pasteur de Paris, le MNHN et l'Inra à propos de l'exploitation des collectes de tiques sur la végétation basse pour les années 2008 et 2009 [...]. On n'a pas accès au matériel brut tant qu'il n'y a pas publication. Enfin, je ne pense pas qu'ensuite on aura accès au matériel brut, mais si déjà on a les informations moulinées par Pasteur sur les huit sites d'études que nous avons en commun, nous ça nous suffit largement » (géographe du Ladyss).

Les écologues peuvent ignorer France Lyme et le géographe, car ces acteurs sont des «outsiders » encore peu reliés à d'autres experts : "Je suis quelqu'un qui, par racro, est arrivé assez récemment sur la maladie de Lyme» (géographe du Ladyss). Cette exclusion est d'autant plus aisée que les écologues ont une excellente maîtrise du lieu d'investigation. Ils sont aussi associés à des acteurs forts, comme Epia et Bipar, laboratoires bien dotés en ressources humaines et en techniques coûteuses 
(notamment des élevages de tiques, avec ou sans animalerie), dont les membres sont également investis dans plusieurs instances (groupe TMT, Centre national d'expertise vectorielle $\left[\mathrm{CNEV}^{7}\right]$, Haut conseil de la santé publique [HCSP], etc.). En revanche, les écologues ne peuvent occulter le CNR, acteur puissant par sa fonction et sa mobilité (les responsables du CNR participent au CNEV, au groupe TMT, au HCSP, etc.). Le CNR était également à l'époque hébergé à l'Institut Pasteur de Paris, qui coordonne l'axe «maladies infectieuses, parasitaires et nosocomiales émergentes » des programmes Picri. Or, en nouant une double collaboration avec les écologues et les géographes, le CNR les a indirectement mis en relation : «Ce qui est plus problématique, c'est que le CNR a cautionné la manip des géographes, $c^{\prime}$ est-à-dire qu'ils se sont positionnés comme intervenants. Ça, c'est une faute à mon avis. Ils ont voulu jouer sur les deux tableaux et il faut choisir » (écologue du MNHN). S'ils n'ont pu exclure le CNR de leurs collaborateurs, les écologues n'en sont pas moins devenus critiques vis-à-vis de leurs études, comme je le montrerai dans la section suivante.

La rivalité qui s'est instaurée entre les géographes et les écologues à propos d'un espace (Sénart), d'une thématique (la probabilité de contracter la maladie de Lyme dans différents milieux forestiers) et de deux bailleurs de fonds (la Région Île-de-France et l'ONF) témoigne d'enjeux relatifs à la répartition des territoires de recherche. Cette rivalité est d'autant plus forte qu'à plusieurs égards, leurs approches se rejoignent, sans néanmoins se superposer. Cette tension s'est établie à partir d'un critère moral (la propriété des idées) dont découle une posture (la confiance), indispensable pour nouer des collaborations. En tant qu'acteurs incontournables, les écologues spécialistes du tamia ont en partie la main sur les collaborations qui s'instaurent à Sénart. Ils ont donc pu se passer d'acteurs «faibles", peu reliés et peu mobiles, mais pas de ceux qui occupent une position centrale. Ainsi, sur cet espace-frontière traversé de rivalités, se sont investis des acteurs suffisamment indispensables pour que malgré tout des collaborations s'établissent entre des acteurs rivaux: le CNR et, dans une moindre mesure, $l^{\prime} \mathrm{ONF}$, qui finance régulièrement les études relatives au tamia mais n'en assiste pas moins celles des géographes.

\section{Configuration 1 : les « outsiders »}

\section{L'écart méthodologique comme signe d'isolement}

Bien que le CNR collabore avec ces deux groupes rivaux, et par voie de conséquence avec leurs partenaires respectifs, les informations issues de la collaboration

7 Porté par les ministères de l'Agriculture et de la Santé, le CNEV a été créé en 2011 pour fournir rapidement une expertise vectorielle aux autorités. Son siège se situe à Montpellier. entre le MNHN, Epia et Bipar ne sont pas partagées avec les géographes et donc non insérées dans leur protocole : «Il a besoin de nous pour traiter l'aspect maladie de Lyme en forêt de Sénart. Nous, on n'a absolument pas besoin de lui. Donc il essaie par tous les moyens de nous soutirer des informations qu'on ne veut pas lui donner puisqu'il nous a fait un coup de tordu " (écologue du MNHN). N'ayant pu bénéficier de la collaboration d'acteurs plus anciennement impliqués dans la problématique des maladies et des tiques, les géographes ont dû élaborer leur propre protocole de terrain. Leur isolement relationnel se note donc par un écart méthodologique.

Contrairement à la majorité des acteurs impliqués dans l'étude de la maladie de Lyme, ils ne récoltent pas les tiques au drapeau ${ }^{8}$ et ne piègent pas les animaux puisqu'ils n'ont pas les compétences nécessaires. Pour accéder aux animaux porteurs de tiques, les géographes se sont associés aux fédérations de chasse qui mettent à leur disposition les chevreuils fraîchement tués. Cette association les confronte à des contraintes que n'ont pas les chercheurs qui récoltent au drapeau ou capturent des animaux : leur temps de travail est soumis aux exigences de la chasse (la découpe de la viande destinée aux boucheries doit se faire rapidement) et ils ne peuvent accéder aux tiques qu'en automne et en hiver, lorsqu'elles sont peu nombreuses. La méthode ne permet donc pas d'évaluer le risque acarologique pendant les périodes printanière et estivale durant lesquelles les tiques sont les plus actives, tout comme les usagers de la forêt. Les géographes tentent bien d'extrapoler le nombre de tiques à ces périodes, mais leurs échantillons sont tellement faibles que cette extrapolation a peu de valeur statistique.

« Ça faisait rigoler les chasseurs parce qu'on chassait des tiques à une période où il n'y en avait pas. Le problème, c'est qu'eux, ils ne chassent qu'à une période où il n'y a pas de tiques et qu'on est obligé de s'adapter à eux [...]. Lors des trois dernières collectes, le mieux qu' on ait pu faire $c^{\prime}$ est trois tiques sur un chevreuil. Et à la dernière, on en a eu quatre pour treize chevreuils. Donc c'est inexploitable» (géographe du Ladyss).

Bien plus que les tiques et leur portage en pathogènes, ce sont les trajets des chevreuils combinés à leur charge en tiques qui intéressent les géographes. Ils veulent en effet établir une carte du risque qui superpose à ce premier

\footnotetext{
8 Concrètement, il s'agit de tirer un drap (de nature et de dimension variable) sur une surface et, à intervalles donnés, de le retourner et d'y récolter les tiques accrochées à l'aide d'une pince. Cette méthode peut être appliquée de façon aléatoire (par exemple, pour estimer une densité) ou sur des zones de forte concentration (pour récolter un maximum de tiques, dans un but expérimental). Malgré l'abondante littérature qui l'entoure, la méthode n'a pas encore été standardisée et chaque équipe l'applique donc « à sa manière ».
} 


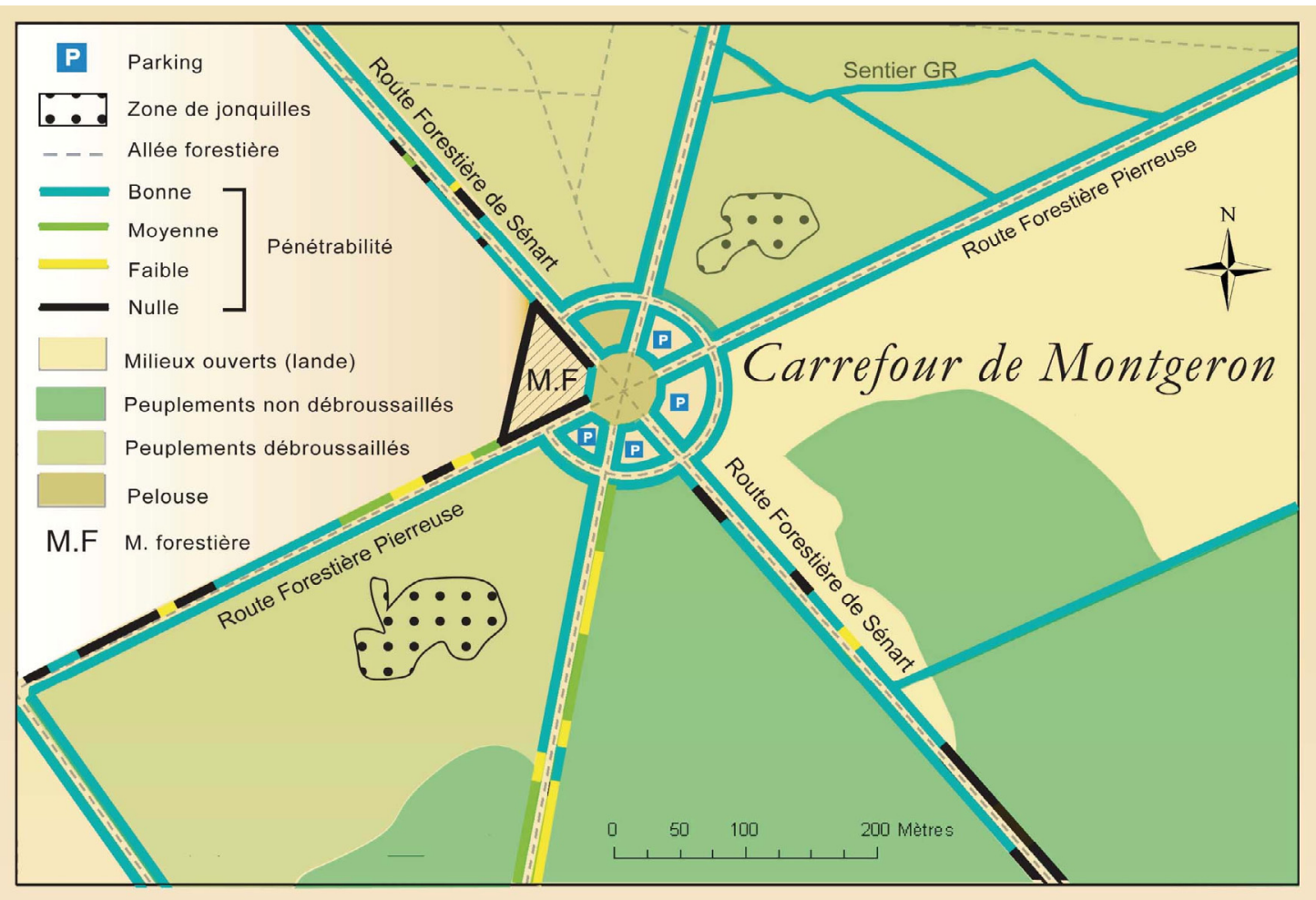

Fig. 1. Représentation de l'espace selon les géographes (carte issue de l'article de Méha et al., 2012).

paramètre acarologique un second paramètre plus anthropologique: les déplacements des promeneurs dans la forêt. Pour eux, la forêt se représente donc comme un espace que traversent plusieurs vivants, selon des parcours que les géographes supposent un minimum réguliers, et dont la rencontre provoque le risque infectieux. Ainsi, comme le montre la carte présentée sur la Figure 1, l'espace est représenté en fonction de son accessibilité aux usagers de la forêt. Y figurent les chemins balisés et leur pénétrabilité, les zones de parking, mais également les types de végétation et leur attractivité. C'est donc l'émergence de pratiques et de comportements à risques qui intéresse surtout les géographes.

\section{L'influence des conflits antérieurs et l'exclusion des partenaires civils}

Le projet Picri sur la borréliose de Lyme, conçu et déposé par le géographe du Ladyss en association avec le CNR et un infectiologue du CHU de Villeneuve SaintGeorges, tente donc de qualifier l'émergence de cas de Lyme en forêt de Sénart. Pour ce faire, les partenaires doivent corréler et cartographier différents paramètres : la densité respective de chevreuils et de tiques qu'ils portent, le taux d'infection des tiques et celui de la population humaine alentour, ainsi que les trajets des chevreuils (donc des tiques) et ceux des usagers de la forêt, dont la rencontre détermine le risque de transmission infectieuse.

Les projets Picri exigent l'implication d'un partenaire associatif. Le géographe a choisi l'association de malades France Lyme, qui était particulièrement visible sur Internet. En insérant France Lyme, le géographe a réuni deux partenaires dont l'antagonisme s'est clairement manifesté au sein du groupe TMT et ailleurs. France Lyme conteste en effet les assertions diagnostiques et thérapeutiques émises par le CNR. Pour l'association, la maladie de Lyme est sous-diagnostiquée et donne lieu à des développements chroniques non résolus par les huit semaines d'antibiotiques préconisées. Sa prévalence serait donc bien supérieure aux chiffres produits par le CNR.

Réticent à l'idée de cosigner un projet de recherche avec France Lyme, le CNR a finalement adhéré au Picri du fait du degré minimal de collaboration établi avec l'association. En effet, les seuls contacts noués entre l'association et les autres partenaires ont été médiés par le géographe coordinateur et se sont limités à l'échange de mails et d'appels téléphoniques. L'association s'est vue exclue des réunions organisées entre scientifiques durant lesquelles la teneur du message préventif et informatif a été définie, sans la consulter, alors qu'elle est normalement chargée d'assurer cette partie. Exclure dès l'origine un partenaire dans la conception d'un projet unissant 
plusieurs communautés de recherche entrave l'hybridation des pratiques, elle-même source d'innovation et d'évolution (Mork et al., 2008). Ce faisant, il est impossible de produire une définition collective de l'émergence incluant la question des causes, qui divise le CNR de Pasteur et France Lyme. Il est à noter également que cette exclusion s'écarte des pratiques actuelles qui tendent à inclure les associations de malades très tôt dans les programmes de santé.

Dans le programme Picri, l'absence de réelle collaboration susceptible de produire un cadrage partagé provient d'acteurs puissants (CNR de Pasteur) qui ne souhaitent pas intégrer le point de vue de l'association. L'obtention des crédits de recherche a néanmoins été conditionnée par la participation de France Lyme, qui bénéficie, par ce biais, d'une forme de pouvoir. Malgré cela, France Lyme passe bien après le CNR de Pasteur, institution puissante que le coordinateur géographe a tout intérêt à ménager, eu égard à son isolement thématique. L'objet intermédiaire qui résultera des collaborations en témoigne, puisque les éléments retenus dans la carte du risque définiront les causes de l'émergence sans prendre en compte celles qui sont importantes pour l'association, la diversité des pathogènes et de leurs souches notamment.

Le programme Picri révèle un problème général relatif à l'hégémonie de certains acteurs académiques qui ont le pouvoir de hiérarchiser la valeur et la pertinence des savoirs produits. En effet, il ne suffit pas qu'un programme de recherche exige l'implication des citoyens pour que ceux-ci soient effectivement considérés par leurs partenaires académiques. Dans le cas du projet Picri, l'association a plutôt été instrumentalisée et Sénart n'opère pas comme un espace-frontière. Les facteurs institutionnels qui ont suscité l'implication de France Lyme ont en effet été contrecarrés par d'autres facteurs institutionnels : la prédominance du CNR dans la thématique de la maladie de Lyme.

\section{Configuration 2 : l'acteur entrepreneur et l'acteur institutionnel}

\section{De la récolte des tiques comme facteur de tension...}

Les écologues du MNHN se sont mis à récolter les tiques au drapeau depuis qu'ils collaborent avec Epia et le CNR, tous deux accoutumés à cette méthode. Concrètement, à la vitesse d'un homme qui marche sans se presser, ils tirent le drapeau sur dix mètres, soit dix longs pas. Ils retournent ensuite le drap et y comptent les tiques, à l'exception des larves, normalement non porteuses de bactéries. Ils réitèrent l'opération seize fois, en séparant chaque relevé de vingt à trente mètres.
Dans un premier temps, il s'agissait d'alimenter les analyses du CNR, de fournir des données à Epia et d'obtenir une valeur de référence dans le milieu, nécessaire pour mettre en relief le parasitisme des tamias. Pour ce dernier objectif, la récolte des tiques est inutile, leur comptage suffit. En plus de servir les études des écologues, la méthode du drapeau leur a permis de développer de nouveaux travaux. En corrélant la densité de tiques dans le milieu à la densité des cervidés, des tamias, et aux types de végétation, les écologues ont pu proposer à la Région Île-de-France une carte des zones à risques de contraction de la maladie de Lyme dans la forêt de Sénart. S'ils n'en étaient au départ pas spécialistes, ces derniers ont donc su tirer parti des récoltes au drapeau, qu'ils mettent désormais en œuvre pour leurs propres projets.

En dressant une carte du risque fondée sur les récoltes de tiques, les écologues se sont approprié une méthode pratiquée de longue date par le CNR et introduits sur son terrain «épidémiologique ». Des tensions relatives à la manière de penser l'épidémiologie se sont alors créées autour des récoltes, chaque partie estimant interroger de manière adéquate le milieu à risques. Progressivement, un désaccord latent sur la paternité de la méthode et ses modalités de mise en œuvre est ainsi apparu entre les membres du MNHN et du CNR. Alors que les membres du CNR estiment l'avoir enseignée aux écologues, ces derniers affirment qu'ils l'ont apprise d'Epia. De plus, étant simple et largement décrite dans la littérature (bien que non standardisée), la méthode ne nécessitait pas $\mathrm{d}$ 'apprentissage par des pairs. Les écologues ont cependant dû s'adapter aux demandes du CNR et modifier légèrement leur protocole, sur un point qui peut paraître de détail mais ne l'est pas, à savoir le nombre de relevés à partir desquels les résultats seront statistiquement extrapolés à l'échelle du massif forestier : «Le seul truc qui a changé c'est qu'on faisait quinze tirages, quinze échantillons, et le CNR en fait seize. Voilà ce qui a changé dans notre protocole depuis qu'on les connaît. On en fait seize parce qu'ils ont décidé que c'était bien, mais je n'en suis pas forcément certain » (écologue du MNHN).

La circulation des tiques récoltées et des analyses concomitantes implique que la méthode soit standardisée 9 . Cette standardisation se heurte aux questions différentes que se posent les chercheurs ${ }^{10}$, aux manières tout

\footnotetext{
9 Cette standardisation est aussi un enjeu dans le groupe TMT dont plusieurs membres pratiquent les récoltes de tiques, sans s'y prendre de la même manière, et sans pouvoir échanger un matériel dont la récupération est coûteuse.

${ }^{10}$ La pluviométrie peut exprimer les mêmes différences entre les pratiques du CNR et des écologues : le CNR ne récolte pas les tiques lorsqu'il pleuvine, estimant qu'elles s'accrochent mal au drapeau, alors que les écologues ne se soucient guère de ce paramètre car ils sont moins intéressés par les tiques que ne l'est le CNR.
} 
aussi variées qu'ils ont d'y répondre et à une forme de pouvoir quant à celui qui parviendra à imposer ses normes, et donc à faire s'aligner les autres chercheurs (enjeu de la standardisation). À cet égard, la question du nombre de relevés est éloquente car elle met en jeu la crédibilité des études sur le mode spécifique des sciences de terrain (Kohler, 2002). À partir de combien et de quels relevés peut-on estimer que les données sont représentatives du lieu?

\section{... à la représentativité des études}

« Pour une forêt de 3200 hectares, le CNR fait huit sites de prélèvement qu'il tire au sort avec une méthodologie [...]. La forêt est tellement hétérogène que huit sites, ça n'a pas de sens. Ils vont avoir huit résultats effectivement, mais de là à pouvoir dire "J'ai une image du massif forestier", évidemment non ! [...] Et donc là, forcément, on n'est pas d'accord » (écologue du MNHN).

C'est sur cette même question de la représentativité que l'écoépidémiologiste de l'Inra et les écologues du MNHN distinguent leur épidémiologie de celle du CNR : « Le CNR a pour objectif d'avoir une idée du risque de la maladie de Lyme en France alors que nous, notre travail, c'est de comprendre comment circule la bactérie entre les tiques et les réservoirs » (écoépidémiologiste de l'Inra). Ils critiquent tout d'abord les échantillons forestiers choisis par le CNR, sans égard pour les différents peuplements. L'idée sous-jacente est que chaque peuplement fonctionne comme un écosystème spécifique, doté d'une faune, d'un degré d'hygrométrie, etc., qui se répercutera sur le risque de maladie : « Plus un milieu est fermé, plus on a de chances d'avoir des densités de chevreuils ou de sangliers. Dans un peuplement de conifères, tu n'as rien » (écologue du MNHN). Étant aléatoires, les sites d'études du CNR ne rendraient pas compte de la diversité et de la spécificité des milieux qui sont au cœur des études écologiques: "On fait des échantillonnages aléatoires pour avoir, sur le plan statistique, une estimation qui soit fiable et on fait au moins deux ans de suite la même région parce qu'il y a des variations interannuelles très importantes » (CNR).

À l'inverse du CNR, les écologues réalisent de très nombreux relevés sur des peuplements choisis (sites) pour mieux représenter la diversité de la forêt, la répartition de la faune sauvage notamment : «On va couvrir toute la forêt. S'il faut qu'on fasse 300 points, un point pour dix hectares, on mettra quinze jours à dix personnes, mais on fera 300 tirages pour un mois donné. Donc on va se donner les moyens d'intégrer tout ce qui est de l'hétérogénéité » (écologue du MNHN).

À travers le nombre de relevés et le choix des peuplements, les écologues critiquent finalement les étapes qui

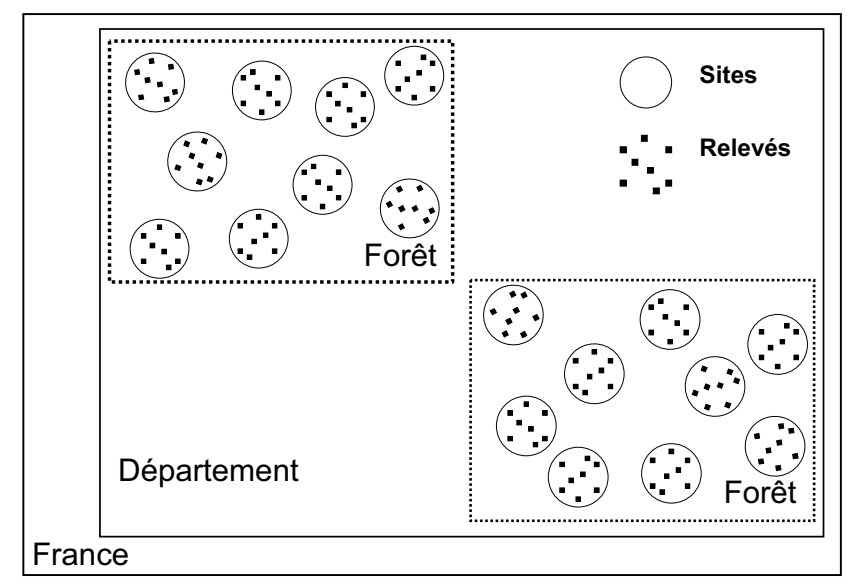

Fig. 2. Représentation de l'espace selon le CNR.

conduisent le CNR à généraliser ses résultats en opérant des transitions entre échelles (du très local au très général) : sur la base de deux massifs forestiers divisés en huit sites aléatoires étudiés pendant deux ans, le CNR extrapole ses résultats à l'échelle d'un département ( $c f$. Figure 2). « Le CNR choisit une forêt et il a un système d'échantillonnage et finalement, ça représente ce qu'ils échantillonnent " (écoépidémiologiste de l'Inra) ; "Le CNR va dire: "Sur les massifs forestiers, ça se passe comme ça" et moi, je dirai : "Sur les huit sites étudiés, ça se passe comme ça". Voilà la différence » (écologue du MNHN). Pour les écologues, les études du CNR sont peu représentatives car elles occultent la singularité des milieux. Cette critique méthodologique exprime aussi un conflit de connaissance : les écologues connaissent Sénart dans ses moindres recoins. Sensibles à son contexte (faune, végétation, usagers), ils accordent aux éléments qui la distinguent plus d'importance que le CNR qui travaille sur toute la France, en accordant peu de temps à chaque forêt. Pour les écologues, le CNR manque donc d'un sens du terrain, d'une connaissance fine du contexte que Kohler désigne par l'expression "residential knowledges » (2012). En agissant comme si tous les lieux se valaient, comme si le terrain n'était pas singulier, il prolonge des pratiques de laboratoire jugées inadéquates par les écologues qui tirent une grande partie de leurs connaissances du rapport familier au terrain et aux techniques appropriées d'échantillonnage.

Contrairement au CNR, ceux-ci capturent les tiques et les tamias en distinguant, au sein de Sénart, trois zones qui représentent chacune un milieu spécifique (cf. Figure 3). Dans la zone centrale, la présence permanente du garde de l'ONF permet d'étudier l'influence des pratiques humaines sur les populations de tamias et de tiques : « Le garde de l'ONF, c'est un élément du paysage. S'il leur donne des graines, ça influence les tamias ». Le deuxième cercle est particulièrement investi par les écologues. Grillagé, le parc de la Faisanderie leur permet en effet 


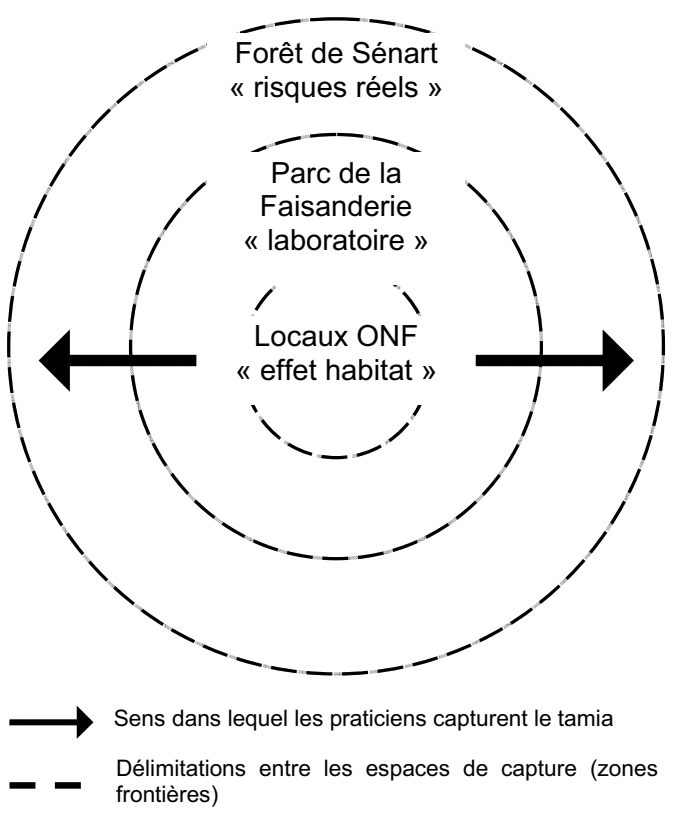

Fig. 3. Représentation de l'espace selon les écologues du MNHN.

d'exclure les usagers humains et leurs compagnons canins, perçus comme des paramètres perturbateurs. Cet espace se rapproche donc des conditions de laboratoire : il limite les variables, c'est-à-dire les entrées/sorties des vivants. Enfin, le dernier cercle est constitué par les alentours immédiats. Cette zone, où le public circule librement, se rapproche des conditions réelles dans lesquelles le risque se manifeste.

La manière dont les chercheurs récoltent les tiques illustre finalement leur représentation del'espace. Pour le CNR, la forêt est envisagée comme un milieu indéterminé et investigué sur base de méthodes aléatoires. Pour les écologues spécialistes du tamia, Sénart est un espace singulier qui s'appréhende selon une logique hybride, entre le laboratoire et le terrain. Enfin, pour les géographes, Sénart est un espace « réel » où circulent des vivants dont la rencontre engendre le risque infectieux.

\section{Conclusion}

La compréhension des maladies comme celle de Lyme, qui sont déterminées par la dynamique des milieux naturels, exige la coopération entre différentes communautés scientifiques. Le risque de transmission engage en effet une multiplicité de facteurs, de processus et de vivants qui relèvent de modes de connaissance variés. Avec leurs apports spécifiques, et tout en restant attachés à leur communauté d'origine, différents scientifiques s'emparent du risque de transmission. Pour en appréhender la complexité, ces scientifiques doivent également se rapprocher d'autres communautés qui produisent des savoirs complémentaires.
Dans cet article, j'ai envisagé comme facteur de collaboration un terrain partagé entre plusieurs communautés scientifiques. La forêt de Sénart est un espace-frontière dans le sens où les chercheurs (et une association de malades) entrent en relation par son intermédiaire. La coopération passe par des échanges de matériel biologique, de données, de techniques et de savoir-faire. Des formes d'apprentissage et de déplacement se notent chez certains acteurs. Ainsi, les écologues et le géographe ${ }^{11}$ se sont immiscés dans les thématiques sanitaires qui leur étaient étrangères et se sont aussi approprié de nouvelles méthodes. L'apprentissage et l'élaboration d'un cadrage partagé par l'ensemble des acteurs ont néanmoins été entravés par des facteurs déontologiques, épistémiques et institutionnels qui ont en même temps motivé l'établissement des relations. Parce que Lyme est une maladie vectorielle qui implique des animaux réservoirs, les biologistes de l'Institut Pasteur sont aussi confrontés à des compartiments du cycle biologique de la bactérie qu'ils maîtrisent moins ou pas du tout.

La coopération suppose d'abord une déontologie entre les chercheurs. Deux configurations, qui ne coopèrent pas malgré des partenaires communs, se sont ainsi formées autour du site de Sénart pour des raisons déontologiques. Le facteur déontologique a influencé les modes de production des connaissances puisque l'acteur «outsider» a dû élaborer un protocole de récolte des tiques qui diffère de celui des autres et garantit l'évitement physique des acteurs en conflit. Enfin, le conflit s'est étendu aux partenaires, qui sont décriés s'ils ne peuvent être exclus. Les liens matériels (partage de techniques, de projets, etc.) ne garantissent donc pas la collaboration. Des dimensions relationnelles, qui impliquent des normes comportementales, sont à aussi considérer.

Ensuite, lorsqu'ils sont en relation, les chercheurs ne coopèrent pas forcément de façon fructueuse, car leur manière de cadrer les problématiques manifeste une relation différente à la singularité du milieu et à la représentativité des relevés. En établissant une carte du risque à l'échelle nationale, le CNR méconsidère le contexte d'étude et fonde la légitimité de ses relevés sur une logique statistique contestée par les autres acteurs, qui privilégient l'étude d'un site contextualisé et dense afin de comprendre la dynamique de la maladie. Si la pratique du CNR, des écologues et des écoépidémiologistes hybride les logiques de terrain et de laboratoire, ils ne font pas importer les mêmes éléments au sein de ces logiques. Chez les écologues, le contrôle expérimental s'opère en étudiant un même espace relativement isolé mais sélectionné tandis que la logique de laboratoire s'exprime chez le CNR par l'étude de sites choisis aléatoirement à partir

\footnotetext{
${ }^{11}$ Suite à la lecture de ma thèse, le géographe m'a confié qu'il veillerait à l'avenir à mieux intégrer les partenaires dès l'origine du projet.
} 
de calculs statistiques. Les coopérations peuvent donc être entravées par des cadrages différents de la maladie, qui associent des questionnements différents mais aussi des impératifs de gestion différents.

Des tensions se sont également manifestées à travers la récolte de tiques, dont la mise en œuvre a laissé apparaître les questions différentes des chercheurs. La standardisation des méthodes est présentée par Star et Griesemer (1989) comme un facteur de collaboration entre communautés hétérogènes. Dans le cas de la forêt de Sénart, bien que la récolte ait été standardisée entre le CNR et les écologues du MNHN, celle-ci n'a pas engendré de réel apprentissage. Par contre, l'apprentissage s'est manifesté entre l'écoépidémiologiste d'Epia, les écologues du MNHN, ainsi que les parasitologues et microbiologistes de Bipar. Membres du groupe TMT, ces acteurs ont poursuivi l'étude de la forêt de Sénart en y incluant d'autres membres intéressés par les relations entre vecteurs et réservoirs. Au sein du TMT, Sénart est ainsi devenu un site d'étude privilégié à propos duquel les données s'accumulent et circulent. La récolte des tiques étant une tâche fastidieuse et l'accumulation des données sur un même site et sur un temps long étant capitale pour fonder les hypothèses des écologues, plus un terrain est étudié, plus il a de chance de continuer à l'être.

Enfin, indépendamment des facteurs déontologiques et épistémiques, les arrangements institutionnels pèsent sur les collaborations entre acteurs. Conçus pour favoriser l'intérêt public, les appels à projets tendent en effet à imposer un cadrage thématique (celui du risque sanitaire) et institutionnel (l'obligation de collaborer). Leur mise en œuvre privilégie également certains acteurs dont la légitimité institutionnelle est reconnue. C'est le cas du CNR, comparativement à France Lyme. Dans le cas de la maladie de Lyme et du site de Sénart, ces arrangements institutionnels ont finalement stabilisé des options de recherche et des méthodologies qui ont elles-mêmes rendu difficiles (voire impensables) des collaborations entre des réseaux d'acteurs qui sont engagés dans des controverses plus larges.

Sur cet espace-frontière, qui rassemble autant qu'il divise $^{12}$, les dimensions déontologique, épistémique et institutionnelle s'interpénètrent et s'influencent sans qu'il soit possible de déterminer laquelle prédomine. Chacune de ces dimensions a en effet motivé les relations qui se sont nouées autour de Sénart. Réciproquement, elles ont montré des limites qui invalident un réel coapprentissage entre tous les acteurs. Pour être efficaces, les collaborations entre disciplines devraient donc relever

\footnotetext{
12 Comme le soulignent Mauz et Granjou (2013), un espacefrontière ne perdure pas forcément au-delà des rencontres entre acteurs et ne débouche pas forcément sur des pratiques partagées.
}

Reçu le 13 août 2014. Accepté le 15 décembre 2015. d'une pratique scientifique capable de penser en même temps ces dimensions déontologique, cognitive et institutionnelle de la recherche.

\section{Références}

Becker H.S., 1963. Outsiders, New York, The Free Press of Glencoe. Trad. Fr. : Becker H.S., 1985. Outsiders, Paris, A.M. Métailié.

Fontenille D., Jourdain F., Léger E., Perrin Y., 2013. Note relative à la distribution d'Ixodes ricinus en France ainsi qu'aux principaux facteurs susceptibles d'impacter la distribution et l'abondance de l'espèce en France métropolitaine, Montpellier, http://www.sante.gouv.fr/IMG/pdf/2013_distribution_ ixodes_ricinus.pdf.

Gilbert C., Henry E., 2012. La définition des problèmes publics : entre publicité et discrétion, Revue française de sociologie, 53, 1, 35-59.

Granjou C., 2013. Micropolitiques de la biodiversité. Experts et professionnels de la nature, Bruxelles, Peter Lang.

Guston D.H., 2001. Boundary organizations in environmental policy and science: an introduction, Science, Technology $\mathcal{E}$ Human values, 26, 4, 399-408.

Kohler R.E., 2002. Place and practice in field biology, History of Science, 40, 2, 189-210.

Kohler R.E., 2012. Practice and place in twentieth-century field biology: a comment, Journal of the History of Biology, 45, 4, 579-586.

Kohler R.E., 2011. Paul Errington, Aldo Leopold, and wildlife ecology: residential science, Historical Studies in the Natural Sciences, 41, 2, 216-254.

Massart C., 2013. Les processus d'écologisation entre santé et environnement. Le cas de la maladie de Lyme. Thèse de doctorat en sociologie, Saint-Martin d'Hères-Liège, Université Pierre Mendès-France Grenoble, Université de Liège.

Mauz I., Granjou C., 2013. A new border zone in science. Collaboration and tensions between modelling ecologists and field naturalists, Science as Culture, 22, 3, 314-343.

Méha C., Godard V., Moulin B., Haddad H., 2012. La borréliose de Lyme : un risque sanitaire émergent dans les forêts franciliennes ? Cybergeo : Revue européenne de géographie, section "environnement, nature, paysage", 601, http://cybergeo.revues.org/25285, DOI : $10.4000 /$ cybergeo.25285.

Mélard F., 2008. Écologisation. Objets et concepts intermédiaires, Bruxelles, Peter Lang.

Mork B.E., Aanestad M., Hanseth O., Grisot M., 2008. Conflicting epistemic cultures and obstacles for learning across communities of practice, Knowledge and Process Management, 15, 1, 12-23.

Star S.L., Griesemer J.R., 1989. Institutional ecology, “translations" and boundary objects: amateurs and professionals in Berkeley's Museum of vertebrate zoology, Social Studies of Science, 19, 3, 387-420.

Trompette P., Vinck D., 2009. Retour sur la notion d'objetfrontière, Revue d'anthropologie des connaissances, 3, 1, 5-27.

Teulier R., Hubert B., 2008. Des concepts intermédiaires pour la conception collective. Les situations d'action collective avec acteurs hétérogènes, in Mélard F. (Éd.) Écologisation. Objets et concepts intermédiaires, Bruxelles, Peter Lang, 163-186. 